\title{
DESIGN OPTIMIZATION AND THE PATH TOWARDS A 2 MW SPALLATION NEUTRON SOURCE*
}

\author{
J. Wei ${ }^{\dagger}$, M. Blaskiewicz, N. Catalan-Lasheras, D. Davino, A. Fedotov, Y.Y. Lee, N. Malitsky, \\ Y. Papaphilippou, D. Raparia, A. Shishlo, N. Tsoupas, W. Weng, S.Y. Zhang, BNL, USA \\ R. Keller, J. Staples, LBNL, USA \\ J. Stovall, J. Billen, S. Kurennoy, S. Nath, H. Takeda, L. Young, LANL, USA \\ R. Sundelin, JLab, USA \\ A. Aleksandrov, Y. Cho, P. Chu, S. Cousineau, V. Danilov, M. Doleans, J. Galambos, \\ J. Holmes, N. Holtkamp, S. Kim, R. Kustom, D. Jeon, E. Tanke, W. Wan, ORNL, USA
}

\begin{abstract}
The Spallation Neutron Source (SNS) is designed to ultimately reach an average proton beam power of $2 \mathrm{MW}$ for pulsed neutron production. The SNS physics groups analyze the machine performance within the hardware constraints, optimize the accelerator design, and establish the best path towards a $2 \mathrm{MW}$ and higher spallation neutron source.
\end{abstract}

\section{INTRODUCTION}

The Spallation Neutron Source (SNS), presently in its third year of a seven-year construction cycle at the Oak Ridge National Laboratory, is intended to deliver a proton beam of up to $2 \mathrm{MW}$ power to a mercury target for neutron spallation. The accelerator system operates at a repetition rate of $60 \mathrm{~Hz}$ and an average current of $1.6 \mathrm{~mA}$. It consists of an $\mathrm{H}^{-} \mathrm{RF}$ volume source (IS) capable of delivering $65 \mathrm{~mA}$ peak current, a Low-Energy Beam Transport (LEBT) housing a first-stage beam chopper, a 4-vane Radio-Frequency-Quadrupole (RFQ) linac, a Medium-Energy Beam Transport (MEBT) housing a second-stage chopper, a 6-tank Drift-Tube-Linac (DTL), a 4-module Coupled-Cavity-Linac (CCL), a Superconducting RF linac (SRF) of medium $(\beta=0.61)$ and high ( $\beta=0.81)$ cavities (SCL), a High-Energy Beam Transport (HEBT) for diagnostics and collimation, and an accumulator ring compressing the $1 \mathrm{GeV}, 1 \mathrm{~ms}$ pulse to $650 \mathrm{~ns}$ for delivery onto the target through a Ring-to-Target Beam Transport (RTBT) [1]. Table 1 lists major parameters.

\section{DESIGN PHILOSOPHY}

The primary concern of building such a high-intensity accelerator is that radio-activation caused by excessive uncontrolled beam loss can limit the machine's availability and maintainability. Based on operational experiences, hands-on maintenance [2] demands that the average uncontrolled beam loss not exceed 1 to 2 Watts of beam power per tunnel-meter. Uncontrolled beam losses are usually attributed to (1) mismatch upon change of linac structure and lattice; (2) space charge effects including parametric

\footnotetext{
* Work performed under the auspices of the US Department of Energy
}

$\dagger$ wei1@bnl.gov, on joint appointment at ORNL and BNL
Table 1: Key parameters of the Spallation Neutron Source accelerators.

\begin{tabular}{lrr}
\hline & Baseline & Back-up \\
\hline Kinetic energy, $E_{k}[\mathrm{MeV}]$ & 1000 & 975 \\
Uncertainty, $\Delta E_{k}(95 \%)[\mathrm{MeV}]$ & \pm 15 & \pm 15 \\
SRF cryo-module number & $11+12$ & $11+15$ \\
SRF cavity number & $33+48$ & $33+60$ \\
Peak field $E_{p}(\beta=0.61)[\mathrm{MV} / \mathrm{m}]$ & 27.5 & 27.5 \\
$\Delta E_{p}(\beta=0.61)[\mathrm{MV} / \mathrm{m}]$ & \pm 2.5 & $\pm 2.5)$ \\
Peak field $E_{p}(\beta=0.81)[\mathrm{MV} / \mathrm{m}]$ & 35 & 27.5 \\
$\Delta E_{p}(\beta=0.81)[\mathrm{MV} / \mathrm{m}] \quad+2.5 /-7.5$ & \pm 2.5 \\
Beam power on target, $P_{\text {max }}[\mathrm{MW}]$ & 1.4 & 1.7 \\
Pulse length on target $[\mathrm{ns}]$ & 695 & 699 \\
Chopper beam-on duty factor $[\%]$ & 68 & 68 \\
Linac macro pulse duty factor $[\%]$ & 6.0 & 6.0 \\
Ave. macropulse $\mathrm{H}^{-}$current $[\mathrm{mA}]$ & 26 & 32 \\
Linac ave. beam current $[\mathrm{mA}]$ & 1.6 & 1.9 \\
Ring rf frequency $[\mathrm{MHz}]$ & 1.058 & 1.054 \\
Ring injection time $[\mathrm{ms}]$ & 1.0 & 1.0 \\
Ring bunch intensity $\left[10^{14}\right]$ & 1.6 & 1.9 \\
Ring space-charge tune spread & 0.15 & 0.20 \\
\hline
\end{tabular}

halo, non-equipartition in the linac, and a high tune spread in ring; (3) limited physical and momentum acceptance; (4) premature $\mathrm{H}^{-}$and $\mathrm{H}^{0}$ stripping and ring injection foil scattering; (5) magnet field errors, fringe field and misalignments; (6) instabilities (transverse impedances, electron cloud); and (7) accidental loss due to system malfunction (ion source and linac, ring extraction kickers).

SNS carefully addresses the above seven issues by adopting a low-loss design philosophy [3]. Furthermore, foreseen losses are localized to shielded areas. Collimation systems are used at the HEBT, the ring (two-stage), and the RTBT. The beam gap is chopped at the LEBT and the MEBT, and cleaned in the ring.

Much of the emphasis in the design of the accelerator centers on machine flexibility and reliability. The superconducting RF linac allows normal operation with one failed cavity/klystron; the ring is designed with an energy acceptance range of $\pm 5 \%$ (determined by $\mathrm{H}^{0}$ stripping loss) to accommodate linac energy variation; a tuning range of 3 units vertical and 1 unit horizontal aids resonance min- 
imization; a robust injection allows independent horizontal, vertical, longitudinal painting manipulation; an adjustable, two-stage collimation system accommodates variable beam size; and an exchange mechanism delivers instant injection foil replacement. In addition, redundancy is reserved in key areas (e.g. spare cryo-module, magnets, hot power supply, diagnostics, etc).

Finally, the entire facility is designed with the potential to reach an output energy up to $1.3 \mathrm{GeV}$ and an output power higher than $2 \mathrm{MW}$, capable of supplying a second target with beam. The higher energy can be reached by upgrading the superconducting RF cavity gradient and klystron power supplies, and by filling existing unoccupied linac tunnel spaces with up to 9 additional cryo-modules. The ring is capable of accommodating the energy increase and delivering the higher beam power without exceeding space-charge limits by an addition of 2 extraction kickers and a replacement of 2 injection chicane dipoles.

\section{LINAC OPTIMIZATION}

\subsection{Design Choices}

The SRF linac operating at $805 \mathrm{MHz}$ frequency accelerates the $\mathrm{H}^{-}$beam from $186 \mathrm{MeV}$ to top energy. The choice of two types of SRF cavities allows for economic savings and future energy upgrades. The choice of cavity geometric $\beta$ value is based on a smooth transition from the warm section linac, a maximized final output energy, and a comfortable transition from medium- to high- $\beta$ section with tolerance to one cavity failure. We also choose constantgradient, continuous focusing to maximize the accelerating field strength [4].

Considering the tight construction schedule, a moderate peak surface field of $27.5( \pm 2.5) \mathrm{MV} / \mathrm{m}$ is chosen for the medium- $\beta$ cavity. Benefiting from electro-polishing, a higher peak field of $35.0(+2.5 /-7.5) \mathrm{MV} / \mathrm{m}$ is assumed for the high- $\beta$ cavity. In order to reduce uncertainties in RF controls of an ion $(\beta<1)$ beam under Lorentz detuning, microphonics, beam transients and injecting energy offset, we decide to drive each cavity with its own klystron using independent amplitude and phase control.

\subsection{Design Studies and Challenges}

A key challenge in linac performance is to minimize beam emittance growth and centroid jitter in both transverse and longitudinal directions upon ring injection, reducing foil traversal, scattering and radio-activation. The warm DTL operating at $402.5 \mathrm{MHz}$ is less sensitive to vibrational noises than most existing linacs operating at 200 $\mathrm{MHz}$. Nevertheless, great care is taken to isolate and minimize system noises.

Using only two types of cavity $\beta$ for over $800 \mathrm{MeV}$ of acceleration compromises the equipartition law. Potentially, space-charge coupling can cause transverse and longitudinal emittance exchange [5] when the emittance ratio meets resonance conditions. In addition, depending on the level of initial mismatch, space-charge parametric halo may de- velop in the linac. Efforts have been made to reserve an economically affordable large aperture in both warm and SRF sections, and to reserve tunability in the MEBT, CCL and SRF linac.

Effects of higher-order modes (HOM) on the cavities is another issue. Overlapping of beam and HOM spectrum is possible because of the pulsed time structure of the beam and the fact that the beam frequency shifts with variable ring energy and repetition rate (e.g. for some two-target operation scenarios). Fortunately, transverse and longitudinal (beam break-up) instabilities are minor issues for an ion beam in the presence of a cavity-to-cavity frequency spread [7]. HOM dampers are implemented only for the purpose of power dissipation [8].

\section{RING OPTIMIZATION}

\subsection{Choice of Accumulator Ring}

During the first year of construction, a study was performed comparing a full-energy linac with accumulator ring to a rapid-cycling-synchrotron (RCS). As opposed to an RCS, an accumulator ring simplifies the capture process, avoids ramping and RF shielding complications, and avoids magnet errors due to ramping, saturation, and power-supply tracking. The study concluded that, especially due to the stringent beam-loss limit of a 2-MW facility, the required RCS design is technically more demanding and less cost effective [6].

\subsection{Choice of FODO-doublet Lattice}

The optimized SNS ring lattice has a hybrid structure with FODO bending arcs and doublet straight sections [3]. The lattice combines the FODO structure's simplicity and ease of correction with the doublet structure's flexibility for injection and collimation. Injection at a dispersionfree region allows independently adjustable painting [9] in the transverse (with orbit bumps) and longitudinal (with an energy-spreading phase-modulated RF cavity) directions for a robust operation. The $12.5 \mathrm{~m}$-long uninterrupted straight section with a flexible phase advance further improves collimation efficiency.

Working point $(6.40,6.30)$

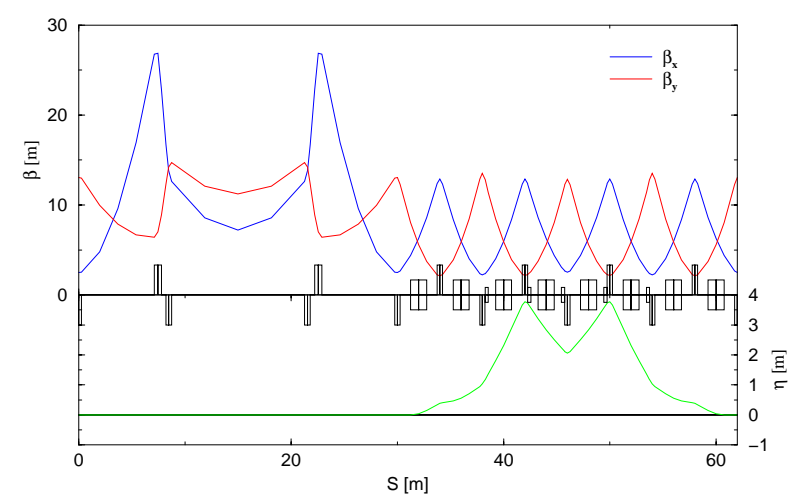

Figure 1: SNS ring lattice super-period of FODO/doublet structure. The machine super-periodicity is 4 . 
Table 2: Beam parameter evolution and an end-to-end simulation example across SNS accelerator systems.

\begin{tabular}{llllllllllll}
\hline & LEBT & RFQ & MEBT & DTL & CCL & SCL 1 & SCL 2 & HEBT & Ring & RTBT & Unit \\
\hline$E_{k}$ (in) & & 0.065 & 2.5 & 2.5 & 86.8 & 185.6 & 387 & 1000 & 1000 & 1000 & $\mathrm{MeV}$ \\
Length & 0.12 & 3.8 & 3.6 & 36.6 & 55.1 & 64.2 & 94.7 & 169.5 & 248.0 & 150.8 & $\mathrm{~m}$ \\
$\epsilon_{n}$ (rms) & $0.09 /$ & $0.19-$ & $0.22-$ & $0.27-$ & $0.29-$ & 0.33 & $0.34-$ & $0.34-$ & $44+$ & $44+$ & $\mu \mathrm{r}$ \\
& 0.2 & 0.21 & 0.27 & 0.29 & 0.33 & 0.4 & 0.56 & 0.59 & 44 & 44 & $\mu \mathrm{r}$ \\
$\epsilon_{u n}(99 \%)$ & & & & & & & & & $120+120$ & $120+120$ & $\mu \mathrm{r}$ \\
$\Delta x$ jitter ( \pm ) & & & & & & & 0.3 & 0.3 & 0.3 & 0.3 & $\mathrm{~mm}$ \\
$\Delta E$ (rms) & & $0.005-$ & $0.007-$ & $0.09-$ & $0.13-$ & $0.19-$ & $0.27-$ & & & & $\mathrm{MeV}$ \\
& & 0.01 & 0.015 & 0.092 & 0.15 & 0.27 & 0.51 & & & & $\mathrm{MeV}$ \\
$\Delta E$ jitter $( \pm)$ & & & & & & & 1.6 & 0.25 & & & $\mathrm{MeV}$ \\
$\Delta E$ (99\%) & & & & & & & 4 & 10 & 10 & & $\mathrm{MeV}$ \\
Codes used & IGUN & PARMTEQ & PARMILA & PARMILA & PARMILA & PARMILA & PARMILA & PARMILA UAL/ORBIT & PARMILA & \\
Loss (control) & 0.28 & - & 0.04 & - & - & - & - & $0-0.001$ & $0.02-0.1$ & 0.04 & \\
Loss (uncont.) & 0.1 & 0.2 & $<0.01$ & $5.7 \mathrm{e}-4$ & $1.4 \mathrm{e}-4$ & $2 \mathrm{e}-6$ & $1.4 \mathrm{e}-5$ & $2.8 \mathrm{e}-5$ & $1.4 \mathrm{e}-4$ & $1 \mathrm{e}-6$ & \\
$\epsilon$ (rms) growth & & 5 & 19 & $18+$ & $12+$ & $14+$ & $3+$ & $0-11$ & 5 & 3 & $\%$ \\
$\epsilon$ (99\%) growth & & & & & & & & $0-20$ & 10 & 5 & $\%$ \\
\hline
\end{tabular}

\subsection{Component Implementations}

The implementation of ring chromatic sextupoles was debated. Sextupoles are not used in rings like ISIS. However, as a higher intensity machine, the SNS requires unprecedented loss tolerance, and relies upon a large momentum spread for instability damping and a large momentum aperture for beam-in-gap/momentum cleaning [10]. Chromaticity sextupoles, powered in four families, are thus essential in avoiding resonances resulting from a chromatic tune spread.

In order to reach a large momentum spread without introducing excessive beam halo, longitudinal painting is implemented. To facilitate such a painting scheme, the output momentum jitter and spread must be strictly controlled by an energy-correcting RF cavity synchronized to the linac frequency at an optimized distance from the end of linac to allow for an adequate beam-phase slippage and a moderate RF voltage. Thus, these "corrector" and "spreader" cavities are also essential.

\subsection{Design Challenges}

The effect of electron-cloud generation [11] imposes a serious threat to a high-intensity ring like SNS. Efforts to address this problem focus two fronts: minimization of electron production, and enhancement of Landau damping. Implementations to minimize electron production includes a pair of tapered magnets for electron collection near injection foil, TiN coated vacuum chamber to reduce multipacting, striped (TiN) coating of extraction kicker ferrite, beam-in-gap kicker to keep a clean beam gap $\left(10^{-4}\right)$, a relatively high vacuum $\left(5 \times 10^{-9}\right.$ Torr $)$, ports screening and step tapering, installation of electron detectors for monitoring, and possible installation of solenoid windings in unoccupied straight sections. Implementations to enhance damping includes a high RF voltage (up to $60 \mathrm{kV}$ ) along with the energy spreader to provide momentum acceptance, lattice sextupole families for chromatic adjustments, and reserved space for possible wide-band damper systems.

\section{END-TO-END SIMULATION}

Extensive efforts have been made to compare and develop models and codes for linac simulations including space charge and SRF, and to develop ring simulation codes (UAL [12] and ORBIT [13]) that handle tracking and mapping along with space charge, painting, magnetic errors, impedances, and collimation. Table 2 is an example of an end-to-end simulation using various codes. The SNS beam loss model is based partly on empirical data at existing facilities and partly on simulations [14].

\section{SUMMARY}

By adopting superconducting RF technology for the linac and by fully optimizing the accumulator ring design, the SNS is following a clear path towards a high-intensity $\left(2 \times 10^{14}\right.$ at $\left.60 \mathrm{~Hz}\right)$, high-power $(2 \mathrm{MW})$ facility.

The authors would like to thank our colleagues of the SNS teams and our collaborators all over the world for their contributions and helpful discussions.

\section{REFERENCES}

[1] R. Kustom, LINAC 2000, p.321.

[2] T. Wangler, RF Linear Accelerators, (Wiley \& Sons, 1998); Workshop on Beam Halo and Scraping, Wisconsin (1999).

[3] J. Wei et al, Phys. Rev. ST-AB 3, 080101 (2000).

[4] J. Stovall et al, LINAC 2000, p.605.

[5] I. Hofmann, et al, these proceedings.

[6] J. Wei et al, EPAC 2000, p.981 and p.123.

[7] R. Sundelin; D. Jeon et al, these proceedings.

[8] S. Kim, et al, these proceedings.

[9] J. Wei, et al, these proceedings.

[10] S. Cousineau, et al, these proceedings.

[11] R. Macek, these proceedings.

[12] N. Malitsky, J. Smith, R. Talman, J. Wei, PAC 1999, p. 2713.

[13] J. Galambos et al, ORBIT User's Manual, 1999.

[14] N. Catalan-Lasheras et al, SNS Notes SNS/AP/7 (2001). 